\title{
Annäherungen an Prometheus
}

Es existieren bei Kafka Texte, die solchem Wunsch wesentlich näher liegen als die im Schloss enthaltene „Legende“ Vor dem Gesetz. Ein ganzer, für uns verpflichtender Textkomplex wurde unter dem 17. Januar 1918 und ohne eigenen Titel im 3. Oktavheft überliefert: „Von Prometheus berichten vier Sagen.“ Franz Kafka hat darunter mehrere Variationen des Prometheus-Mythos aufgereiht. Berichtet wird zunächst einmal über Prometheus' Bestrafung durch Zeus, dann vom Verschmelzen des schmerzgepeinigten Feuerbringers mit dem Felsen, danach einsetzendes allseitiges Vergessen - und schließlich wurde man „des grundlos Gewordenen müde. Die Götter wurden müde, die Adler. Die Wunde schloss sich müde. Blieb das unerklärliche Felsengebirge."1 Das Ende alles organischen Lebens wird derart beschworen. Kafkas Aufzeichnungen geschahen im Zusammenhang mit einer Überschau über weitere zentrale griechische Mythengestalten, als da sind: Die Sirenen, der Meeresgott Poseidon und, wie ausgeführt, jener Feuer, Wärme und Licht bringenden, selbst göttlichen Rebell Prometheus, - was alles in das letzte Jahr des Ersten Weltkriegs fiel, mithin in eine Zeit, in der der Prager bereits wusste, dass „Kakanien” verloren, der Habsburgische Staat, als dessen Beamter er beweisbar identifikatorisch arbeitete,

\footnotetext{
${ }^{1}$ Zitiert nach Franz Kafka, Gesammelte Werke in zwölf Bänden. Nach der Kritischen Ausgabe herausgegeben von Hans-Gerd Koch, Frankfurt/Main 1994 (Fischer Taschenbuch Verlag), Bd. 6, S. $192 \mathrm{f}$.

Nach dieser Ausgabe wird im Folgenden mit wenigen Ausnahmen (etwa FaksimileDrucken) zitiert, in besonderen Fällen aus der noch von Max Brod veranstalteten Taschenbuchausgabe in sieben Bänden, Frankfurt/Main 1976 (Fischer Taschenbuch Verlag).
}

(C) Der/die Autor(en) 2021

B. Neumann, Umrisse einer Dritten Kultur im interdisziplinären Zusammenspiel zwischen Literatur und Naturwissenschaft, ELECTRISCHER PROMETHEUS. Umrisse einer Dritten Kultur im interdisziplinären Zusammenspiel zwischen Literatur und Naturwissenschaft, https://doi.org/10.1007/978-3-662-63204-8_2 
nicht mehr zu retten war. In diese Zeit fiel auch Kafkas intensive und hektische, für ihn vernichtende, will sagen an den Abgrund des Nervenzusammenbruchs bringende Hans-Blüher-Lektüre. ${ }^{2}$ Blüher, dieser vormalige Prophet der Jugendbewegung, so etwas wie ein alter und vertrauter Buch-Bekannter Franz Kafkas, verkündete zu Weltkriegsende immer lauter und besessener die Beendigung der jüdischen Assimilation; und der Prager nahm den Reichsdeutschen als einen klassisch gelehrten Zeitdiagnostiker ganz erstaunlich ernst. Gemeinsam war Kafkas damaliger Beschäftigung immer eines: Ihre Gegenstände hatten einen Glanz verloren, den sie zuvor ganz offenbar und unbestreitbar noch besessen hatten. Und das unvorstellbar plötzlich. In schlagartig erfolgter Entropie, wie Blumenberg ausführt. Die Sirenen singen nun nicht mehr; der vormalige Meeresgott Poseidon, eingeschworener Feind des Odysseus, agiert gar als subalterner Rechnungsführer mit Ärmelschonern und hat seinen Dreizack verloren; selbst ein Prometheus bringt keine Wärme mehr zu den Menschen, sondern verwächst mit seinem Felsen zu einer (am Ende gar nicht mehr organischen) grau erstarrten Einheit, nachdem, das immerhin, seine Leber-Wunde, die Strafe des Zeus, sich „müde“ geschlossen hatte. Damit wird keine Heilung angezeigt, sondern darauf verwiesen, dass selbst das Wundgeschehen erstarrt. Und Hans Blüher, der vormaliger glühende Verehrer dionysischer Lebendigkeit und Gegner allen apollinischen Masshaltens, verkündete seinerseits das Ende der jüdischen Assimilation!

In vieler Hinsicht kann man also hier von Kafkas "Arbeit am Mythos“ sprechen. Als kein Zufall erscheint, dass in Hans Blumenbergs (inzwischen selbst zu mythischem Ruhm gelangten) Titel Arbeit am Mythos die Kafka'schen Prometheus-Variationen ihrerseits zum Thema gemacht werden. Dies geschieht in elektrisierender Weise. Nämlich als eine Diagnose vom Tod des Mythos selbst - eine Diagnose, die bei Blumenberg zudem naturwissenschaftlich abgeleitet erscheint aus dem Zweiten Hauptsatz der Thermodynamik. Der besagt ja, dass die Entropie geschlossener Systeme notwendigerweise zunimmt, was laut dem von Blumenberg bemühten Zweiten Hauptsatz der Thermodynamik dazu führt, dass diese kollabieren, mithin notwendig der Entropie verfallen. Diesen Zweiten Hauptsatz sieht der Gelehrte auch gespiegelt in Sigmund Freuds 1920 erfolgter Einführung des Todestriebs. Letzterer werde nun, auch das ein Resultat des ernüchternden Weltkriegserlebnisses, der Libido entgegengesetzt ,als das große

\footnotetext{
${ }^{2}$ Vgl. dazu Bernd Neumann, Aporien der Assimilation, München (Fink) 2007, S. 210 ff.
} 
Modell, dem ... Freud das Organische und Psychische ... unterworfen hat, als er, in seinem dem Mythischen sich immer mehr näherndes System, 1920 den Todestrieb einfügte." 3 Für den Kafka der Prometheus-Variationen aber ginge es darum, wenn schon nicht den Mythos, so doch einen Mythos zum Verlöschen zu bringen, diesen endgültig in die Vergessenheit zu befördern. Und in der Tat insistiert der einstige Blutsbruder von Kleists „electrischer“ Schöpferbegeisterung jetzt nur noch darauf, mit tief erkältender Monotonie zudem, dass alles organische Leben eben sein Ende finden müsse - in einem universalen entropischen Todesgeschehen. Ein Vorgang, der von dem völlig desillusionierten Prager im Herzen der prometheischen Feuer-Mythe selbst angesiedelt wird: Als endgültiges Erlöschen einer einst flammend hellen Menschheitshoffnung. Alles ist vorbei. Für immer. „Die Götter wurden müde, die Adler wurden müde, die Wunde schloss sich müde" - diese Kafka-Sätze zitiert auch Hans Blumenberg. Ferner auch die abschliessende Sentenz: „Die Sage versucht das Unerklärliche zu erklären. Da sie aus einem Wahrheitsgrund kommt, muss sie wieder im Unerklärlichen enden." Damit schliesst sich ein Kreis, kehrt die Schöpfung zu ihrem Ursprung zurück, zu einer mineralischen, kalten und erstarrten Welt ohne jedes Feuer, ohne Wärme, ohne Licht, ohne Elektrizität, darum ohne Hoffnung, ohne Leben. Blumenberg nennt Kafkas epochalen Text deshalb eine ,großartige und rücksichtslose Imagination“, ${ }^{4}$ gerade weil in ihm die ,alte“ Antithese von Mythos und Logos, von Prähistorie und Historie, gar von Barbarei und Kultur selbst aufgehoben, ja umgedreht erscheine. Mithin in eine Befindlichkeit verwandelt, in der alles Bestehende nur noch als Vergangenes erscheint. Wo nur noch Anorganisches die ehemals lebendig-organische Geschichte, sie ist nun selbst irreparabel verunglückt in die eigene allumfassende Versteinerung hinein, zu überstehen vermochte. Lediglich Mineralisches erscheint jetzt noch als dauerhaft. Blumenberg also liest Kafkas Prometheus-Variationen durchaus ,naturwissenschaftlich" - will sagen, im Licht der unvermeidbar kommenden, dann alles vernichtenden Entropie gemäss dem Zweiten Hauptsatz der Thermodynamik (den er, wie gesagt, noch einmal gespiegelt sieht in Freuds Einführung des „Todestriebs“ vom Jahr 1920). Ein „Endspiel“ sei so in Kafkas Text entstanden; freilich nicht aus existentialistischem ennui inspiriert, sondern durch, wie Blumenberg meint,

\footnotetext{
${ }^{3}$ Hans Blumenberg, Arbeit am Mythos, Frankfurt/Main 1979 (suhrkamp taschenbuch wissenschaft 1805), S. 688.

${ }^{4}$ Hans Blumenberg, Arbeit am Mythos, a. a. O. S. 688.
} 
sogar physikalisch gewonnene Einsicht in den unumgänglichen Entropie-Tod des gesamten Universums.

Das alles bei Weltkriegsende, und während der Prager sich seine Bedrückungen durch Hans Blühers antisemitische Wende notiert in einer epochal zutreffenden Ahnung: "Noch spielen die Jagdhunde im Hof, aber das Wild entgeht ihnen nicht, so sehr es jetzt schon durch die Wälder jagt. " ${ }^{5}$ Ein einziges apokalyptisches finis austriae wird hierin imaginiert. Die von Kafka so genannten „Berichtigungen“ des Prometheus-Mythologems korrigieren also konsequent jene Emphase, die Kafka, als den „Blutsbruder“ Kleists, einst zum selber „electrischen Prometheus“ gemacht hatte. Hans Blumenberg, die führende Autorität auf dem Feld der metaphorischen Vermittlung zwischen Weltsicht und naturwissenschaftlicher Erkenntnis, leitete dies in Arbeit am Mythos aus dem Kampf des „Fortschrittsoptimismus in der zweiten Hälfte des 19. Jahrhunderts mit dem physikalischen Gegenprinzip des Wärmetods" ab. ${ }^{6}$ Er rekurriert mithin auf einen Kafka, der seine Weltsicht, bemerkenswerterweise, aus der Begegnung mit der neueren Physik (und nicht nur der Maxwells oder Einsteins) gewonnen haben sollte. Das Feuer brachte einst die Wärme, daraus entstand auch Licht, das dann als Wechselstrom die Welt eroberte noch vor dem Beginn des dann alles wieder vernichtenden Weltkriegs. Im übrigen etwas, was die Pariser Weltausstellung von, ausgerechnet, 1900 hatte erstrahlen lassen im Lichte der damals neu entwickelten, selbst quasi gottgleichen Generatoren aus dem Hause Siemens. Eben jenen Vorgang hatte bereits der junge Romanautor Kafka in seinem Verschollenen fasziniert verfolgt. Das zentrale Thema erst Maxwells, dann später des jungen Einstein (und, wie gesagt, auch das des ebenfalls noch sehr jungen Kafka im Verschollenen) war eben immer - das Licht mit seiner unübertrefflichen Geschwindigkeit gewesen. Der Verschollene als ein Lichtroman und Einsteins Beschäftigung mit dem Licht als Fortsetzung von Maxwells elektromagnetischen Feldversuchen: All diese Errungenschaften der jüngsten naturwissenschaftlichen Zivilisation würden mit der Niederlage des Habsburgerreiches, mit seinem Verschwinden aus der Geschichte, im „Gegenprinzip des Wärmetodes“ enden, wie Blumenberg es formuliert hat ${ }^{7}$. Und so war es dann ja auch gekommen, nach der verblüffenden, elektrisierenden und noch höchst lebendigen Symbiose des frühen Franz Kafka mit seinem mesmeristischen „Blutsbruder“ Heinrich von

\footnotetext{
${ }^{5}$ Franz Kafka, Gesammelte Werke, a. a. O., S. 181 (s. Anmerkung 38).

${ }^{6}$ Hans Blumenberg, Arbeit am Mythos, a. a. O., S. 688.

${ }^{7}$ Hans Blumenberg, Arbeit am Mythos, a. a. O., S. 688.
} 
Kleist, - worüber nun zu berichten sein wird, als über eine ganz besondere Osmose zwischen archaisch antikem Titanenkampf einerseits, und ganz modernem, gar feministischem Jiu-Jitsu andererseits.

Open Access Dieses Kapitel wird unter der Creative Commons Namensnennung 4.0 International Lizenz (http://creativecommons.org/licenses/by/4.0/deed.de) veröffentlicht, welche die Nutzung, Vervielfältigung, Bearbeitung, Verbreitung und Wiedergabe in jeglichem Medium und Format erlaubt, sofern Sie den/die ursprünglichen Autor(en) und die Quelle ordnungsgemäß nennen, einen Link zur Creative Commons Lizenz beifügen und angeben, ob Änderungen vorgenommen wurden.

Die in diesem Kapitel enthaltenen Bilder und sonstiges Drittmaterial unterliegen ebenfalls der genannten Creative Commons Lizenz, sofern sich aus der Abbildungslegende nichts anderes ergibt. Sofern das betreffende Material nicht unter der genannten Creative Commons Lizenz steht und die betreffende Handlung nicht nach gesetzlichen Vorschriften erlaubt ist, ist für die oben aufgeführten Weiterverwendungen des Materials die Einwilligung des jeweiligen Rechteinhabers einzuholen.

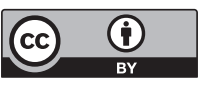

